\title{
Photovoltaics blooms and spreads
}

\author{
The growth of photovoltaics in electricity markets and in research laboratories brings exciting challenges in scaling- \\ up innovative technologies and deploying them for a variety of applications.
}

$\mathrm{R}$ enewable energy is changing the electricity supply in many parts of the world. In 2017, renewable sources contributed $25 \%$ of electricity generation worldwide ${ }^{1}$, taking advantage of globally installed capacities of more than 1,200 GW for hydropower ${ }^{2}, 510 \mathrm{GW}$ for wind power ${ }^{1}$ and about $400 \mathrm{GW}$ for solar photovoltaics by the end of the year ${ }^{1}$. As is well documented in this journal and many others, each of these energy sources has its own advantages and drawbacks.

One of the most impressive features of photovoltaics is its versatility. Because photovoltaics is made up of small elementary units, called solar cells and modules, it can be deployed at small and large scales, from portable electronics to solar farms reaching gigawatt scales. At utility scale, the priority is increasing the amount of generated electricity while minimizing its cost; mainstream technologies such as silicon are the workhorses to achieve this goal. However, the variety of potential applications for solar photovoltaics generates a diversity of technical requirements on devices and makes room for alternative materials and device layouts. For example, the weight requirements imposed by some emerging applications such as portable charging or ground transportation lead to innovations in thin photovoltaic materials and protective layers. In this Focus issue, we point the spotlight on some recent efforts to scale-up photovoltaic technologies from the laboratory to the real world and explore current developments in the deployment of photovoltaics.

Indeed, scaling-up the fabrication of small-area solar cells into larger-area ones and then assembling several of these cells into a solar module is far from trivial. Frequently, a gap in the photoconversion efficiency appears between the solar cell and the module scales. Thin film

$\mathrm{Cu}(\mathrm{In}, \mathrm{Ga})(\mathrm{S}, \mathrm{Se})_{2}$ photovoltaic devices offer potential cost advantages and comparable solar cell efficiencies relative to silicon photovoltaics. However, the module efficiencies are still low - $\mathrm{Cu}(\mathrm{In}, \mathrm{Ga})(\mathrm{S}, \mathrm{Se})_{2}$ photovoltaics exhibit a large efficiency gap relative to scaled-up technologies, namely silicon or CdTe. In their Review, Veronica Bermudez from Solar Frontier (previously at EDF R\&D) and Alejandro Perez-Rodriguez from the Catalonia Institute for Energy
Research and the University of Barcelona discuss the mechanisms causing the $\mathrm{Cu}(\mathrm{In}, \mathrm{Ga})(\mathrm{S}, \mathrm{Se})_{2}$ efficiency gap and outline the solutions developed in academic and industrial research laboratories over the past decades to try to overcome the issue.

Taking a photovoltaics technology from the laboratory to the real world also requires a good understanding of how it behaves in real operating conditions, for example when subjected to weather, and how it evolves with time. Indeed, the expected lifetime of a product under specific use conditions will dictate whether and how it can be deployed. Much work has gone into understanding the many degradation pathways of various photovoltaic technologies and standardized tests to assess the reliability of these technologies when deployed in the field are under constant development. In their Perspective, Henry Snaith from the University of Oxford and Peter Hacke from the National Renewable Energy Laboratory describe the methodology used to develop these standardized tests, discuss their current shortcomings and argue that the lessons learned from this experience can inform studies of operational stability in novel photovoltaic technologies at the pre-commercial stage. However, they also highlight that the priority in the early stages of development is to understand degradation pathways in realistic operation conditions. As such, they review the currently known degradation mechanisms of metal halide perovskite-based photovoltaics, which are now moving into pre-commercial development.

Maximizing and maintaining the photoconversion efficiency is a primary research focus in photovoltaic research. However, the efficiency is only one of the many factors directing if and how a specific photovoltaic technology may be used in a specific application. Minimizing the overall cost of photovoltaic electricity is a primary driver, in particular at utility scale. The cost of photovoltaic electricity does not only depend on the module efficiency and cost: it also depends on the amount of electricity that can be generated per unit area of the installation and on the cost of land, installation, electronics and so on. The cost of photovoltaic electricity can therefore be lowered using these other parameters. For example, bifacial modules can harvest light from both sides, which improves the module power output while keeping the module cost almost constant. In their Comment, Radovan Kopecek and Joris Libal from the International Solar Energy Research Center Konstanz argue that while bifacial modules are already being deployed, the major challenge to be overcome for a true large-scale roll-out of bifacial photovoltaic technologies is to lift the uncertainty on the exact energy yield to be expected from each specific bifacial installation.

Alternatively, for some applications, factors other than cost and efficiency may dominate and need to be optimized for, such as weight for portable applications or aesthetics for integration into buildings or urban landscapes. In their Comment on the integration of photovoltaics in buildings, Ballif and co-workers from the Ecole Polytechnique Fédérale de Lausanne and the CSEM-Swiss Center for Electronics and Microtechnology argue that photovoltaic products are reaching a stage of development and tailorability where they can now be considered as construction materials from the initial stages of the building design, rather than as mere electronic components. The multidisciplinary team discusses how coordinated research and development efforts across the photovoltaics value chain, including engineers, architects and construction experts, are currently changing the way we perceive and implement building-integration.

Indeed, scale-up and deployment of any photovoltaic technology depends on a large network of actors with different skills and priorities, from students in research laboratories to professionals in private companies who ensure that the best technological solutions for each local context are implemented and managed in the field. While this Focus issue of Nature Energy is by no means an exhaustive picture of photovoltaics research and development, we hope it illustrates the exciting variety of endeavours regarding scale-up and deployment, and that it can help cross-pollinate ideas between communities.

Published online: 8 June 2018 https://doi.org/10.1038/s41560-018-0185-1

References

1. Global Energy \& CO2 Status Report 2017 (IEA, 2018).

2. Renewables 2017: Hydropower (IEA, 2017); https://www.iea.org/ topics/renewables/hydropower 